\title{
APLICACIÓN DE MAPAS DE TÓPICOS AL ANÁLISIS SEMÁNTICO DE ALGUNAS COMEDIAS DE CALDERÓN
}

\author{
Miriam Peña Pimentel \\ The University of Western Ontario \\ Department of Modern Languages and Literatures \\ University College, $\mathrm{Rm} 115$ \\ London, ON Canada N6A3K7 \\ mpenapie@uwo.ca
}

[Anuario calderoniano (ISSN: 1888-8046), 5, 2012, pp. 115-130]

Este artículo es un primer acercamiento a la aplicación de mapas de tópicos en estudios filológicos y es parte de un proyecto mayor que plantea el uso de Nuevas Tecnologías aplicadas a las Humanidades. Para este trabajo utilizamos una pequeña selección de comedias calderonianas que forman parte de la Primera parte de comedias de Calderón de la Barca: Casa con dos puertas, El sitio de Bredá y El príncipe constante. Esta selección se basa en la aparición cronológica de las Partes y no necesariamente en la cronología de las comedias y dado que el enfoque de este estudio es presentar una aproximación metodológica, se decidió acotar los sujetos de estudio para da únicamente una muestra de la utilidad del modelo. El trabajo que aquí se presenta se desarro- 
1la en cuatro apartados: comenzamos presentando una introducción a la herramienta teórica manejada (los mapas de tópicos); continuamos en el apartado 2 mostrando el uso que hacemos de esta herramienta para el caso concreto que aquí se presenta, donde se incluye el esquema sobre el que desarrollamos el estudio y el método seguido para poblar dicho esquema a partir de las tres obras seleccionadas; en el tercer apartado se presentan los primeros resultados de la investigación; y el trabajo cierra con algunas conclusiones acerca del uso de esta herramienta y sus posibles aplicaciones a un estudio global de Calderón.

\section{INTRODUCCIÓN}

En el transcurso de un estudio filológico es habitual trabajar con grandes cantidades de datos no estructurados pero, simultáneamente, es esencial extraer y mantener la estructura semántica de los textos, es decir, de representar la relación, sentido y significación entre los componentes del texto. Durante estos últimos años se han usado diversas herramientas para ayudar en este problema, pero pocas de ellas se pueden considerar exitosas a la hora de manejar la estructura de datos necesaria y las relaciones semánticas que se precisa mantener entre los mismos.

En este trabajo presentamos una nueva metodología para extraer información filológica de documentos de texto basándonos en ciertos paradigmas nacidos de la futura implementación de la web semántica. En concreto, los mapas de tópicos (Topic Maps) proporcionan la posibilidad de manejar grandes cantidades de información a diferentes niveles de abstracción, manteniendo las relaciones semánticas entre las unidades de datos utilizadas (así como otros tipos de relaciones), y a la vez permiten la introducción de información complementaria (no contenida explícitamente en los textos originales) que ayude a la interpretación de dichos datos.

Para probar la eficacia del método que proponemos aquí, estudiamos una pequeña porción de la producción dramática de Calderón de la Barca, enfocando este estudio en algunas piezas de las Comedias, partiendo de la idea de que Calderón fundamenta sus obras en los preceptos filosóficos y teológicos contemporáneos y presentes en la doctrina de Contrarreforma del siglo XVII. 
Como una primera muestra de esta metodología, nos centramos en el análisis de algunas producciones que se reúnen en la Primera parte de comedias. La selección de las obras que se han analizado se basa en dos principios: primero, la cronología de la colección de Comedias; y segundo, la variedad de géneros y diversidad de herramientas dramáticas que estas obras presentan. Como continuación de este estudio (y que ya está siendo abordado como trabajo actual), se proyecta el mismo análisis sobre el resto de comedias de Calderón, siguiendo el orden cronológico que muestran las Partes, y no necesariamente el de las obras.

En la siguiente sección presentamos el uso que hacemos del concepto abstracto de Mapa de Tópicos, y la adaptación que se ha considerado de sus componentes para aplicar dicho concepto a un estudio filológico de textos clásicos, revisando desde la creación del esquema que soporta la estructura abstracta de las obras hasta el rellenado concreto del Mapa de Tópicos con los datos de las obras seleccionadas.

2. ¿QUÉ SON Y CÓMO SE USAN LOS MAPAS DE TÓPICOS EN NUESTRO TRABAJO?

Los mapas de tópicos son modelos de representación de conocimiento y, debido a que su objetivo principal es el de organizar la información por medio de las relaciones semánticas entre los datos, conceptos y fuentes asociadas, encontramos en ellos una herramienta de gran utilidad para almacenar contenido semántico que es imposible de almacenar en estructuras más simples. Formalmente, la estructura matemática que se usa para la representación de estos mapas es la de un grafo: una colección de nodos que se conectan entre sí por medio de aristas. En este sentido, usaremos los componentes principales de los mapas de tópicos (tópicos, asociaciones y ocurrencias) para proyectar los elementos fundamentales de las obras literarias. Pasemos a una breve descripción de la Herramienta y de sus componentes principales.

Siguiendo la definición de Pepper:

[Topics] can be any «thing» whatsoever - a person, an entity, a concept, really anything - regardless of whether it exists or has any other 
specific characteristics, about which anything whatsoever may be asserted by any means $[\ldots]^{1}$.

Habitualmente, los tópicos se agrupan jerárquicamente por medio de relaciones ontológicas del tipo: «es un». En estos casos, el tópico superior se denomina tópico tipo o tópico clase, y sus «hijos» son llamados instancias. Por ejemplo, podemos considerar un tópico llamado "personaje», que se comportará como un tipo, frente a tópicos como «Pablos Ballón» o «Calabazas», que son instancias del tipo «personaje».

Las Asociaciones establecen una relación entre dos o más tópicos, por ejemplo, representando, una relación semántica entre los datos. La mayor parte de la información semántica almacenada en un mapa de tópicos recae en las diferentes asociaciones que se usen en su representación. Desde un punto de vista práctico, para construir el mapa de tópicos, establecemos las asociaciones según el contenido semántico que extraemos del texto a partir de la interacción de los tópicos que se reconozcan en el mismo. En nuestro caso concreto, las asociaciones pueden ser las acciones que relacionan un personaje con una actividad, las reacciones de los personajes, las conexiones entre los diversos tipos definidos y sus instancias, etc. Al igual que ocurre con los tópicos, las asociaciones pueden ser agrupadas según un tipo de asociación que, por lo general, se determina por su función dentro del texto. Por ejemplo, la asociación «Justino Nasau habla del Conde de Vergas» (que relaciona las dos instancias anteriores) es del tipo "hablar_de», que determina un nivel más alto en la jerarquía del mapa relacionando, en este caso, dos tópicos que son instancias del tipo «Personaje».

En la teoría general de mapas de tópicos, una ocurrencia es un recurso externo que se relaciona con los tópicos y/o asociaciones. Para nuestro objetivo, además de otros recursos que puedan ser de interés para el análisis filológico y no se encuentren dentro de la obra anali-

1 «In fact, this is almost word for word how the topic map standard defines subject, the term used for the real world "thing" that the topic itself stands in for [...] Strictly speaking, the term "topic" refers to the object or node in the topic map that represents the subject being referred to. However, there is (or should be) a one-toone relationship between topics and subjects, with every topic representing a single subject and every subject being represented by just one topic. To a certain degree, therefore, the two terms can be used inter-changeably» (Pepper, 2000, p. 9). 
zada, usamos las ocurrencias para hacer referencia a los fragmentos de las obras que corresponden a la asociación o tópico concreto del que se trate. Así, cada fragmento de la comedia del cual extraemos una asociación entre diversos tópicos se vuelve una ocurrencia tanto para la asociación extraída como para los tópicos que intervienen. Por ejemplo:

Tópico instancia del tipo «Personaje»: Don Manuel Asociación Instancia del tipo «Asociación»: hablar_de

Tópico instancia del tipo «Lugar»: Escorial

Asociación resultante: Don Manuel hablar_de Escorial

Ocurrencia extraída del texto: «Si solo a Palacio fuera / estuviera más de espacio / pero mi afán inmortal / mayor término ha pedido: / su Majestad ha salido/ esta tarde al Escurial, / y es fuerza esta noche ir / con mis despachos allá / que de importancia será» [La dama duende, J. II, vv. 319-327].

En la figura 1 podemos ver la interfaz del sistema usado para almacenar los diversos componentes del mapa de tópicos asociado a las obras analizadas.

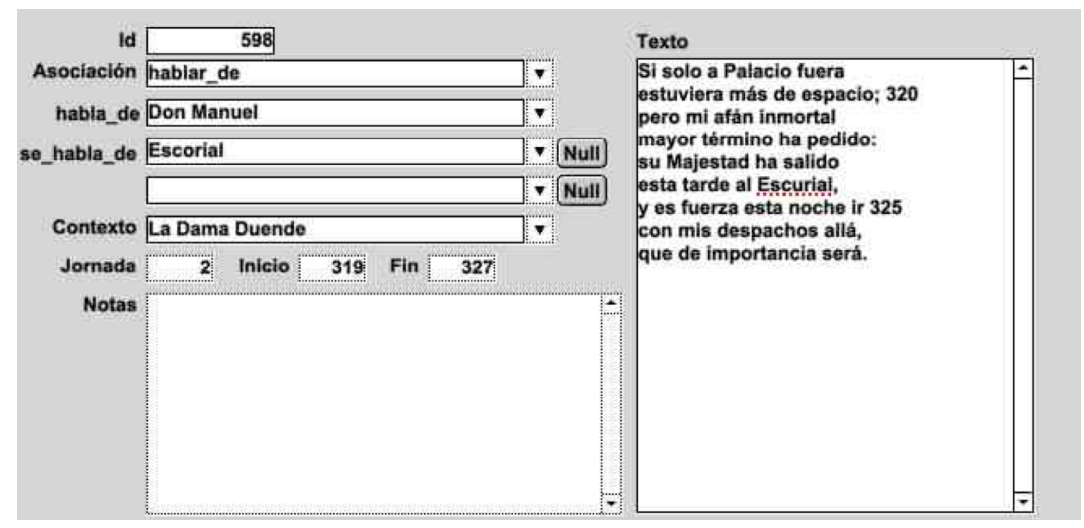

Figura 1. Ejemplo de asociación con personajes y ocurrencia

Como se ha comentado, con el fin de enriquecer más el contenido del mapa que se construye y darle un contenido que permita su uso general como herramienta de investigación, también añadimos 
como ocurrencias información suplementaria del resto de los elementos que forman nuestro mapa, como puede ser: artículos de investigación relacionados con los elementos, referencias externas acerca de un concepto, etc. Tal y como ocurría con los tópicos y las asociaciones, las ocurrencias también pueden ser agrupadas en tipos para proporcionar una organización jerárquica; por ejemplo, considerando el tipo de recurso externo que es (revista, libro...), el formato físico en el que está publicado (papel, digital, oral...), etc.

Si realizamos un corte en el nivel superior de la jerarquía de elementos que definen un mapa de tópicos (los tipos de tópicos, los tipos de asociaciones que hay entre ellos y los tipos de ocurrencias que complementa su información) vemos que se obtiene una representación abstracta de cómo está siendo organizada la información que se extrae del texto. Habitualmente, este nivel abstracto se organiza de manera independiente de sus instancias, y se denomina esquema del mapa, permitiendo obtener un mapa de tópicos organizado en dos capas diferenciadas: la capa del esquema y la capa de datos (o instancias).

Además de los elementos básicos que se consideran en los mapas de tópicos, y que han sido descritos en los párrafos anteriores, para este estudio usamos un concepto adicional de la misma teoría que permite enriquecer aún más la estructura resultante. Es lo que se conoce como contexto (scope). Como su nombre indica, un scope es el contexto en el cual una relación es válida (se considera como relación tanto las asociaciones entre tópicos, como las relaciones que se pueden dar con las ocurrencias). Por ejemplo, «los celos causan violencia» puede ocurrir en el contexto de una de las obras, pero no en el contexto de las demás. De este modo, por ejemplo, para nosotros cada una de las obras representa un contexto, que valida (o no) las diversas asociaciones que se pueden dar entre los tópicos. Pero no sólo consideramos contextos determinados por obras, sino que añadimos posibles relaciones que se pueden dar entre los mismos tópicos de forma adicional a la obra. Por ejemplo, la asociación «Madrid está en España» se da en un contexto denominado como "geográfico" y que es independiente de la obra analizada.

De esta forma, conseguimos contextualizar la información de cada obra, pudiendo hacer uso de los mismos tópicos en muchas de ellas, y sin perder la clasificación intrínseca que el análisis de dichas obras proporciona. 


\subsection{El ESQUema DEL TM}

De un análisis filológico preliminar, extraído de una primera lectura de las obras, podemos construir la versión inicial del esquema con sus tipos de tópicos principales: personajes, lugares, acciones, sentimientos y objetos. Este esquema proporciona la estructura sobre la que se basará la capa de datos, y contiene los primeros tipos de tópicos y de relaciones semánticas en forma de tipos de asociaciones.

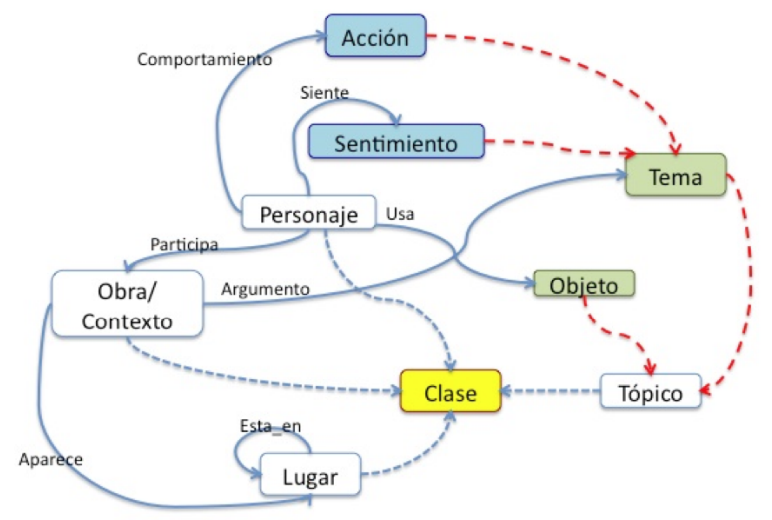

Figura 2. Diagrama del esquema general sobre el que se construye el mapa de tópicos

\subsection{Rellenado Del MAPA DE TÓPICOS}

Dado que tenemos tipos de tópicos que son comunes a todas las obras, creamos para ellos un contexto "general» al que se asocian. Puesto que las asociaciones extraídas del análisis de las obras están directamente relacionadas con las obras concretas de las que se extraen, construimos contextos separados para cada una de estas obras, donde toman parte sus respectivas asociaciones. Así, en nuestro caso, disponemos de un contexto "general» y un contexto para cada una de las obras analizadas. Además de las asociaciones de cada contexto/obra, existen asociaciones independientes de éstas, como pueden ser las asociaciones geográficas o genealógicas, que no corresponden necesariamente a una obra concreta; para éstas, creamos los contextos adecuados a los 
que poder asignar estas asociaciones. Algunos ejemplos pueden ser «contexto geográfico" (en el cual podemos encontrar asociaciones como: "Cádiz está en España»), "contexto histórico» ("Felipe IV fue rey de España»), "contexto mitológico» («Marte es el dios de la guerra»), etc.

El proceso de rellenar las asociaciones extraídas de una obra específica (recuérdese que en este caso cada obra se considera un contexto) requiere el empleo de anotación manual y un análisis de cada obra para especificar la semántica de la relación existente entre los tópicos involucrados. Como ya indicamos, añadimos como ocurrencia a esta asociación el fragmento de la obra de la cual se extrae (en nuestro caso, indicamos, además, la jornada de la obra a la que corresponde y el conjunto de versos que corresponden al fragmento), teniendo en cuenta que, por lo general, de un mismo fragmento obtenemos distintas asociaciones de varios tipos.

Debido a la complejidad de los textos literarios, que muestran diferentes niveles de interpretación (psicológico, histórico, retórico, etc.), actualmente no es posible realizar este rellenado de forma automática, ya que los avances en el área del Procesamiento de Lenguaje Natural (PLN) todavía no lo permiten. Es por esta razón que, en nuestro caso, se hace necesario el proceso manual de rellenado, siendo el investigador responsable del análisis que requiere dicho proceso; es decir, para crear cada una de las anotaciones se requiere de un análisis y conocimiento previo del tema, además del soporte obtenido por medio de estudios críticos sobre teatro del Siglo de Oro y sobre Calderón en particular. A pesar de los problemas que ello conlleva, creemos que esta metodología sigue siendo interesante por hacer evidentes relaciones semánticas que, de otra forma, no serían apreciables por un análisis estándar automatizado.

En el caso de las comedias, debido a la numeración de los versos, las asociaciones aparecen inherentemente asociadas a una distribución lineal o temporal. Esta distribución «temporal», junto con el grafo obtenido de las conexiones, proporciona una representación espacial de las obras que puede ser usada para realizar mediciones cuantitativas y comparaciones entre obras distintas. Por ello, entre las opciones que se abren a partir de este estudio destacamos la de medir la complejidad de las obras por el número de diferentes líneas temáticas simultáneas en el «tiempo", la importancia de ciertas líneas argumentativas en un obra particular por su reiteración y conexiones con otras líne- 
as presentes, o el valor objetivo de algunos tópicos presentes en los discursos de las obras, ya sea por el número de apariciones que tiene en la obra, por el número de conexiones con otros tópicos fundamentales en la trama o su aparición en un conjunto importante de obras. En la figura 3 se muestran los grafos obtenidos de las tres obras analizadas, donde se pueden observar los tópicos más relevantes durante el transcurso de la trama (nodos con mayor número de conexiones), y las asociaciones más comunes (conexiones más gruesas).

Figura 3.Visualización de los mapas obtenidos de las 3 obras por separado:

a) Casa con dos puertas; b) El sitio de Bredá; y c) El Príncipe constante 


\section{Algunos resultados del uso de esta herramienta}

La información recopilada durante los procesos de anotación semántica de los textos se puede agrupar de diversas formas: podemos considerar que cada obra, junto con los contextos necesarios para su interpretación, conforman un mapa de tópicos independiente (como se muestra en la figura 3), o considerar el mapa completo obtenido del análisis semántico de un conjunto de obras. En la figura 4 se puede observar el mapa obtenido de las tres obras aquí analizadas.

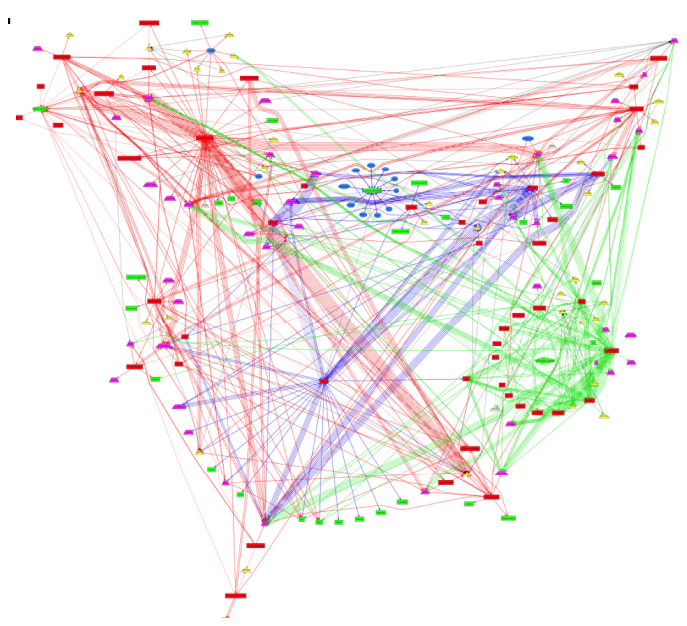

Figura 4.Visualización del mapa de tópicos con los 3 contextos

De la representación semántica de las obras en forma de mapa de tópicos podemos extraer varias conclusiones que pueden ayudar a enriquecer un análisis filológico de las mismas. En este primer acercamiento resultó evidente que el análisis más directo que se puede aplicar al mapa de tópicos que se obtiene da cada obra por separado, consiste en estudiar las frecuencias de aparición de los tópicos y las asociaciones que en él intervienen, determinando aquellos que destacan por su peso en el conjunto global de frecuencias y proporcionando una interpretación filológica a ese hallazgo. A modo de ejemplo, en la figura 5 presentamos los resultados de un análisis exhaustivo del tipo de participaciones que los personajes de Casa con dos puertas realizan a lo largo de la obra. 


\section{Participación de los Personajes}

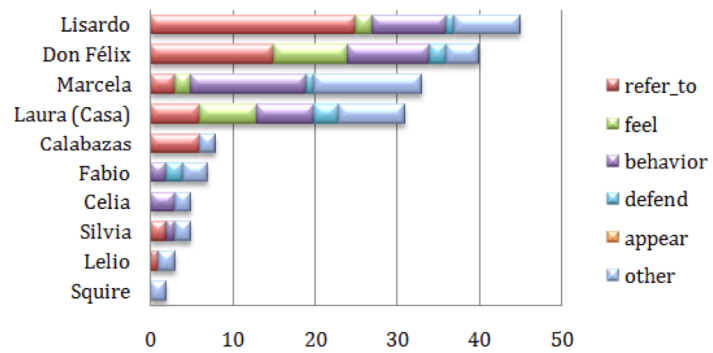

Figura 5. Análisis de la participación de personajes en Casa con dos puertas

De igual modo, tras unir los tres contextos en un mapa de tópicos global (figura 4), encontramos los tópicos y las asociaciones que por su frecuencia de aparición en el grafo resultante nos permiten establecer un punto de partida para un análisis semántico de conjuntos de obras y, con ello, abren la posibilidad de rastrear algunas «temáticas sobresalientes» en la producción calderoniana. Bien es cierto que esta muestra cubre una pequeña parte de esta vasta producción, pero al ser parte de un proyecto más grande, es un indicio que nos ayuda a mapear los tópicos relevantes comunes en esta producción. En la figura 6 mostramos el gráfico correspondiente al análisis de frecuencias aplicado al conjunto de las tres obras analizadas en este trabajo.

\section{Frecuencias de Tópicos}

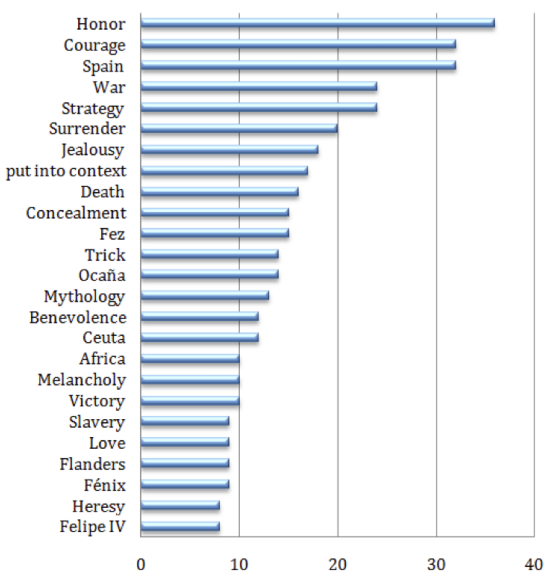




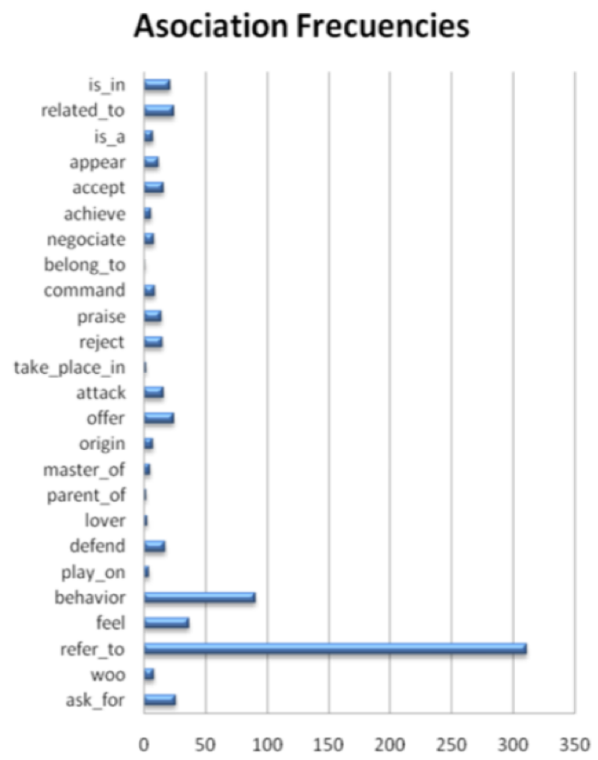

Figura 6. Análisis de frecuencias (tópicos y asociaciones) en el conjunto de las obras

Es importante hacer notar que, a pesar de que muchos resultados parecen ser cuantitativos o que el peso que juegan en el análisis recaiga, en parte, en la frecuencia de aparición en la obra, este procedimiento se diferencia de los habituales en minería de textos en la medida que ésta no conserva el sentido y significado de los textos, sino en un análisis de la frecuencia de aparición de términos sintácticos, ignorando, en general, el contenido semántico de los componentes de la obra y las relaciones que se dan entre ellos y que es lo que mantiene el sentido de la obra.

\section{CONCLUSIONES}

Como resultado del trabajo realizado aquí, podemos concluir que el uso de mapas de tópicos proporciona una herramienta suficientemente robusta para realizar estudios en textos complejos. La producción teatral del Siglo de Oro y en especial la de Calderón comprenden textos de una complejidad temática y estilística que requieren de 
un manejo masivo de información, al mismo tiempo que es imprescindible mantener, de manera estructurada, las relaciones semánticas que se desarrollan a lo largo de cada trama.

Además, de la estructura obtenida haciendo uso de los mapas de tópicos obtenemos también la posible extracción de diferentes visualizaciones de las obras, con la posibilidad de mantener en todas ellas la referencia al texto original. Ya hemos visto en las secciones anteriores las visualizaciones que se obtienen en forma de grafo (ya sea individualmente para cada obra, o para el global de información recopilada). En la figura 7 se puede observar una representación de la misma información haciendo uso del carácter lineal al que hicimos mención anteriormente, abriendo nuevas posibilidades de estudio y de interpretación de los resultados.

Finalmente, creemos que el uso que proporciona esta nueva tecnología en el campo de las humanidades en general, y en el del análisis filológico en particular, abre la posibilidad de acercar los estudios filológicos al creciente uso de medios automatizados, y sin duda agiliza el estudio de producciones globales permitiendo el manejo de grandes cantidades de información que antes eran inabarcables para los investigadores. Es importante resaltar el hecho de que, a diferencia de lo que ocurre con otras aproximaciones que hacen uso de análisis digital, en la metodología que aquí presentamos el trabajo del filólogo se hace imprescindible, ya que, solamente, tras el análisis de los textos se puede producir un mapa de tópicos de una riqueza que no puede ser comparable a la obtenida por medios absolutamente mecánicos.

Por supuesto, debe indicarse que esta metodología no tiene porqué reducirse al análisis de obras puntuales de un autor, sino que puede ser aplicada para abordar estudios más genéricos que permitan analizar y comparar géneros literarios, producciones completas de autores, épocas estilísticas, etc.

Como trabajo futuro nos planteamos continuar con el desarrollo de la herramienta que permite aplicar los mapas de tópicos al análisis de textos, así como también cubrir la mayor cantidad posible de la producción dramática de Calderón y generar diferentes visualizaciones que nos ayuden a comprender la producción calderoniana y formar una sección del que puede ser un mapa cultural del Barroco reflejado en sus obras. 


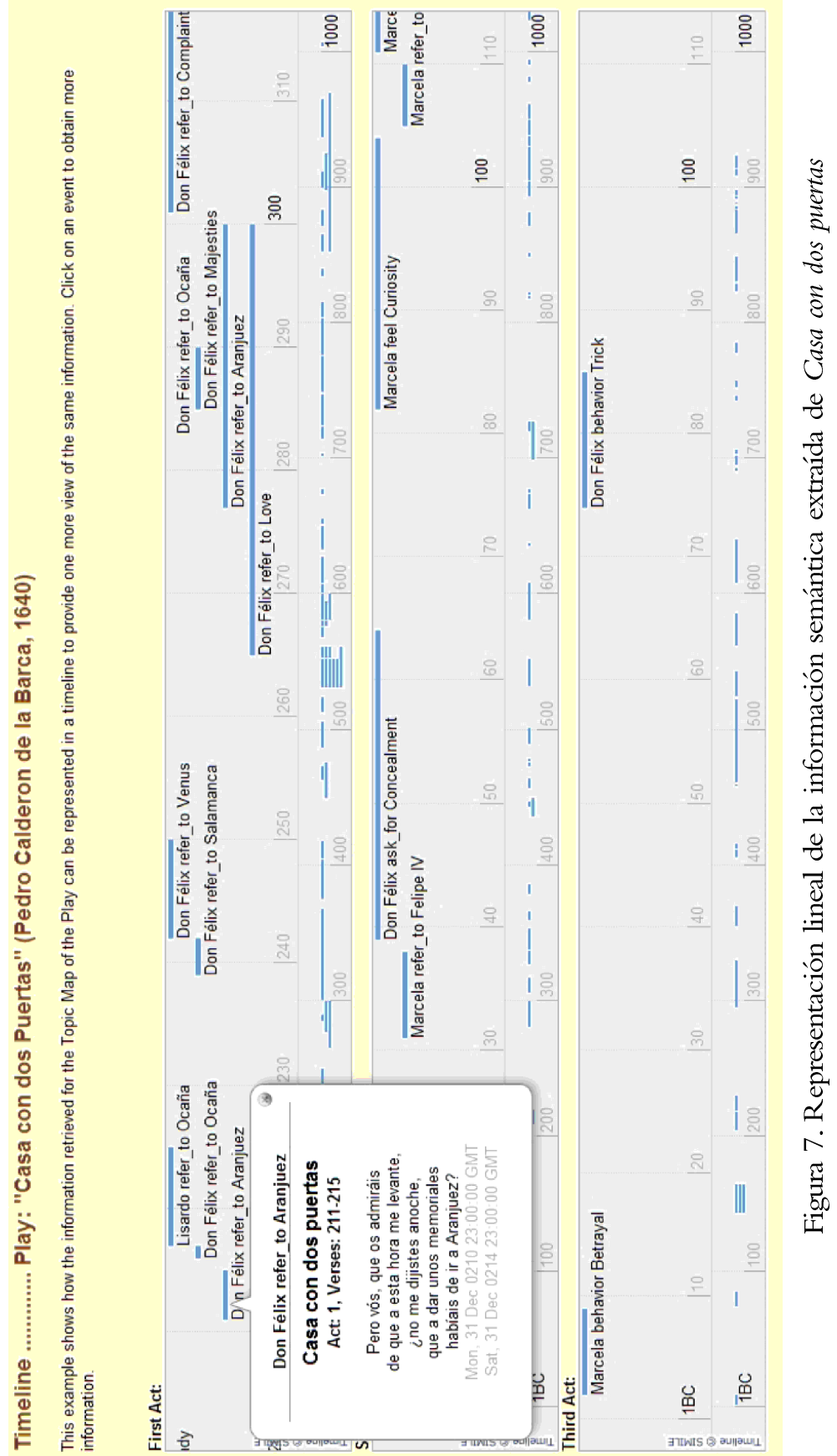




\section{BiBLIOGRAFÍA}

Arellano, I., Calderón y su escuela dramática, Madrid, Ediciones del Laberinto, 2001.

- y Godoy, E., (ed.), Temas del barroco hispánico, Madrid / Frankfurt, Iberoamericana / Vervuert, 2004.

BöHm, K. y MAicher, L., «Real-Time Generation of Topic Maps from Speech Streams», en TMRA 2005, LNAI 3873, Berlin, Springer-Verlag, 2006, pp. 112-124.

Calderón de la Barca, P., El sitio de Bredá, ed. 1640, Cervantes Virtual, \ttp://www.cervantesvirtual.com/servlet/SirveObras/1214163531892 0495543213/index.htm?marca $=\mathrm{el} \% 20$ sitio\%20de\%20breda\#>.

- Casa con dos puertas, ed. 1640, Cervantes Virtual, <http://www.cervantesvirtual.com/servlet/SirveObras/91337519109793162900080/index.htm? marca $=$ casa $\% 20$ con $\% 20$ dos $\% 20$ puertas \#>.

- El príncipe constante, ed. 1640, Cervantes Virtual, <http://www.cervantesvirtual.com/servlet/SirveObras/57949430323572941976613/index.htm? marca $=\mathrm{el} \% 20$ principe $\% 20$ constante\#>.

Cruickshank, D. W., Don Pedro Calderón, Cambridge / New York, Cambridge University Press, 2009.

Díez Borque, J. M. ${ }^{a}$, El teatro en el siglo XVII, Madrid, Taurus, 1988.

García Lorenzo, L., "Secuencias de capa y espada: escondidos y tapadas en Casa con dos puertas», en Calderón. Actas del Congreso internacional sobre Calderón y el teatro español del Siglo de Oro, ed. L. García Lorenzo, Madrid, CSIC, 1983, vol. I, pp. 433-449.

Huerta Calvo, J. (coord.), Historia del teatro español, Madrid, Gredos, 2003, vol. I.

Kazienko, P. y Litwin, M., "On Using Topic Maps for Knowledge Representation. Information Systems Applications and Technology», en Seminar. Proceedings of the 24th International Scientific School, Wroclaw, Wroclaw University of Technology, 2003, pp. 100-107.

Kertész, A., Cognitive Science and Scientific Knowledge. Case Studies in the Cognitive Science of Science, Amsterdan / Philadelphia, John Benjamins Publishing Company, 2004.

Leuenberger, M., Grossmann, S., Stettler, N., y Herget, J., «Topic Maps for Image Collections», TMRA 2005, LNAI 3873, Berlin, SpringerVerlag, 2006, pp. 107-111.

Lumsden-Kouvel, A., "A Counter-Reformation Hero: The Saint and Martyr in Calderón's El Príncipe constante», Bulletin of Hispanic Studies, 77.1, 2000, pp. 101-110. 
Moreiro GonzÁlez, J., «De los Teasuros a los Topic Maps: Nuevo estándar para la representación y organización de la información», Encontros Bibli: Revista Electrónica de Biblioteconomía, 18.2, 2004, pp. 1-19.

Park, J. y Cheyer., A., «Just for Me: Topic Maps and Ontologies», TMRA 2005, LNAI 3873, Berlin, Springer Verlag, 2006, pp. 145-159.

Pepper, S., The TAO of Topic Maps: Finding the Way in the Age of Infoglut, <http://www.ontopia.net/topicmaps/materials/tao.html, 2000>.

Ríos y Ríos, A., Biografía del célebre poeta dramático D. Pedro Calderón de la Barca, San José, B. Rueda, 1883.

Rodríguez Cuadros, E., Calderón, Madrid, Síntesis, 2002.

Sánchez, L. y Fernández, N., «The semantic web: Fundamentals and A Brief State-of-the-Art», The European Journal for the Informatics Professional, 6.6, 2005, pp. 5-11.

Sigel, A., Towards Knowledge Organization with Topic Maps.

<http://www.gca.org/papers/xmleurope2000/papers/s22-02.html, $2000>$.

Steiner, K., Ebmayr, W. y Wagner, R., «Topic Maps. An Enabling. Technology for Knowledge Management», en 12th International Workshop on Database and Expert Systems Applications (DEXA 2001), 3-7 September 2001, ed. A. M. Troja, Munuch, IEEE Computer Society, 2001, pp. 472476.

Strosetzki, C., Teatro español del Siglo de Oro: teoría y práctica, Madrid / Frankfurt, Iberoamericana / Vervuert, 1998.

Vosters, S., La rendición de Breda en la literatura y el arte de España, London, Tamesis, 1973.

WANDORA, <http://www.wandora.org/wandora/wiki/index.php.?title=Wan dora $>$.

Wardropper, B., Critical Essays on the Theatre of Calderón, New York, New York University, 1965. 